\title{
Advanced Telemedicine System Using 3G Cellular Networks and Agent Technology
}

\author{
Golam Sorwar ${ }^{1}$ and Ameer $\mathrm{Ali}^{2}$ \\ ${ }^{1}$ School of Commerce and Management, Southern Cross University \\ NSW 2450, Australia \\ golam.sorwarescu.edu.au \\ ${ }^{2}$ Department of Computer Science and Engineering, East West University \\ Dhaka, Bangladesh \\ ameer7302002@yahoo.com
}

\begin{abstract}
The world's ageing population and prevalence of chronic diseases have lead to high demand for healthcare services. Telemedicine systems based on modern information and communication technology are expected to play a pivotal role in alleviating the pressure on health care services. Fortunately there have been a rapid advanced in technologies including wireless communication especially the third generation network (3G), Internet, software agent and health care devices in terms of mobility, speed and communication. However the current systems are limited in terms of mobility, flexibility and privacy issues. More over the existing solution does not provide any seamless integration of various healthcare providers to provide an effective and efficient team-based continuous care services for patients with chronic illness who prefer to stay in a community based setting. In this paper, we present a generic mobile health monitoring system based on $3 \mathrm{G}$ mobile network and software-agent which involves a set of intelligent agents. These software agents will work as human agents in collaborating among different health care professionals for offering team-based medical services. The proposed system can be implemented in a number of situations in a mobile environment.
\end{abstract}

Keywords: Telemedicine, cellular networks, multi-agent system.

\section{Introduction}

Telemedicine is increasingly gaining popularity due to its high potential for cost savings and increased efficiency in healthcare. Expert medical care through telemedicine can significantly improve the health care facilities at rural and remote locations. Advanced wireless and networking technologies coupled with recent advances in biotechnology, biosensors and software engineering has enabled the promising growth in telemedicine health care systems. These advances will have strong impact on some of the current health care services together with reshaping the workflow and practices in the delivery systems of these services [1], [2].

The widespread use and availability of wireless systems and the Internet brought new opportunities for public and healthcare providers to efficiently access the medical 
services and information with enhanced technological tools. Most recent advancements in wireless communications systems, namely $3 \mathrm{G}$ mobile networks and WiMAX broadband access now have the potential to significantly enhance telemedicine services by creating a flexible and heterogeneous network within an endto-end telemedicine framework. The data rates of current $3 \mathrm{G}$ networks are much higher than those of current $2 \mathrm{G}$ cellular systems. Furthermore, the fast growth of mobile systems and the extensive acceptance of cellular technology signify that $3 \mathrm{G}$ mobile phone services are an exciting new application domain for these services.

A number of emergency telemedicine solution, particularly remote monitoring, [3[, [4], [5], [6], [7], [8] have been proposed by adopting this emerging $3 \mathrm{G}$ network. Though these systems provide a promising opportunity, they are very limited in terms of the flexibility and application diversities. More importantly, they offer very limited communication facilities among a diverse group of service providers. In real world, patient management and medical decision making involves collaboration between a number of health professionals such as nurses, general practitioners, specialist consultants, paramedics, the patients and patients' career and social worker. This is particularly true for chronic diseases where the follow up takes place over a long period of time involving a number of people. Moreover, most of these systems do not have ability to process, analysis and inform patient locally; medical data was processed by data processing servers located remotely.

In order to model collaborative decision-making and to provide the opportunity for mobile device to analysis the results and make some recommendation locally, multiagent systems provide a suitable solution. More recently, researchers have successfully attempted at developing systems in health care domains using this approach [9], [10]. However, the proposed systems are specific application oriented. For example, [9] proposes an agent-based e-health hospital information management system to improve the efficiency of the in-hospital healthcare services. Chan [10] proposes a multi-agent based e-heath monitoring framework to improve the doctorpatient interaction spanning multiple remote locations and hospitals. The main drawbacks of this system are: (1) limited video conferencing facilities, (2) not flexible to switch automatically from non-emergence to emergence situation, and (3) no involvement of other healthcare and technical services such as 24-hour emergency service, the out of hours GP services or technician for biomedical devices functional status monitoring. Integration with social services will often be necessary as a significant number of elderly people are also frail and require daily support, or suffer from dementia.

Addressing some of these issues, this paper proposes an advanced telemedicine framework based on the $3 \mathrm{G}$ cellular networks and the software-agent that seamlessly allows a team-based approach in information sharing and collaborating in decision making across different health care sectors, and that offers maximum continuity of care for the patient.

This paper is organized as follows: Section 2 presents possible heath care service scenarios. Section 3 gives an overview of the proposed system whereas Section 4 details the implementation model of the system. Section 5 presents a multi-agent based software model to be used in the proposed system while Section 6 concludes the paper. 


\section{Possible Healthcare Services}

The proposed model is designed with the possible healthcare scenarios or service platforms that are presented in the subsequent sections.

\subsection{General Web-Based E-Medicine and Treatment}

In a web-based e-medicine and treatment service, the patient will be able to log into a website dedicated for this service and be able to access his/her health records together with previous history. In this system, three entities like patient, doctor and the pharmacist will be responsible for the success of this service platform. Their individual responsibilities and interaction with the system is stated below.

Patient: Firstly, a patient will register with this website will his/her full information and then provide the system with following information: (i) regular update of the patient condition, (ii) exercise details, (iii) update some vital parameters in regular basis that are related to patients, (iv) update of medication reactions, and (v) maintain appointment with the doctor for meeting such as video conferencing with the doctor.

Doctor: A doctor associated with a patient will do the followings: (i) regular monitoring of patient record and updates, (ii) updating the medication or treatment with change of the patient condition, and (iii) Updating the prescription for the patient.

Pharmacist: A pharmacist associated with the patient will do the followings: (i) regular monitoring of patient medication updates and (ii) post a confirmation of the availability of new medicines.

\subsection{Home Monitoring}

The senior citizens, who are suffering for chronic diseases, need constant assistance from the doctors and nurses. Moreover, the patients who are being discharged from the hospital early often require additional healthcare services and monitoring of their health status at home. Furthermore, current scenario of shortage of doctors for providing emergency operation to all patients, lack of hospital beds, and insufficient medical facilities in health care sector with the high expense involved in conventional hospital treatments, homecare monitoring is a promising telemedicine solution [11]. Home monitoring consists of two sub-service scenarios: a) regular monitoring and b) emergency monitoring. In regular monitoring system, the vital signs such as ECG, SPO2, and blood pressure can be monitored periodically in non-real-time (NRT) store-and-forward mode from the patient unit (home) to the central patient health record system (PHR) at the hospital while in an emergency home monitoring system, the patients at critical medical condition are treated in real-time (RT) monitoring mode i.e. the patient's vital signs are transmitted to the PHR in RT [12]. If any vital signs will be in alarming condition an alert message will be transmitted to PHR and from the PHR to the doctor unit. The doctor will contact with the nurse or the relatives of the patient with the facility of Video Conferencing, live video, and image transmission. In regular monitoring, if the patient condition becomes critical, the 
monitoring will be converted to emergency mode and hence does all the necessary actions according to emergency mode.

\subsection{Community Care/Rural Health Centres}

Patients at the community care and rural health centres do not often get necessary treatment because of the lack of professional doctors and the high care facilities. Applying telemedicine model, the community care patients can get high care medical support and professional doctor's treatment from the central/developed hospitals and specialist doctors. The services may range from regular treatment to emergency monitoring of patients in RT with the facilities like video conferencing between the doctor at central hospital and nurse/social worker attending the patient in the community care.

\subsection{Pre-hospital Emergency Ambulance Service}

Every year more than thousands of people die in the car accidents. Urgent initial prehospital treatment is crucial for the savings of severely injured people. In a study performed in UK, $91 \%$ of the people who die from cardiac arrest, do so outside the hospital due to lack of immediate treatment [13]. Pre-hospital emergency ambulance service can be seen as possible solution to these problems. In this emergency service, the ambulance should be equipped with necessary equipments for transferring patient data and video in RT mode through the $3 \mathrm{G}$ network from the ambulance to the doctors present in the hospital. The paramedics in the ambulance can initiate video conferencing with the doctors at hospital so that they can get best support and guidance from the specialist doctors in hospitals.

\subsection{ICU Patient Care}

Suddenly, a patient condition in the ICU may become worse. If the doctor is not at the treatment room in order to attend a meeting outside the hospital, in that situation the emergency ICU patient care may cause lifesaving of the patients. In emergency ICU care, the doctor can monitor the patient status from his mobile/notebook/Laptop in RT/NRT mode to give the attending nurse correct instructions for the savings of the patient.

\section{Proposed System Model}

This work aims at providing effective and mobile solutions to all kinds of healthcare needs, discussed in previous section, for both emergency and regular using $3 \mathrm{G}$ networks and Internet. Therefore, it is essential to develop an integrated, compact, reliable and easy to use telemedicine system which is able to provide all kinds healthcare supports that is needed to rural or remote patients outside the hospital ranging from simple regular health check-up to emergency care to the patients together with real time monitoring and care from distance.

This section presents a newly proposed telemedicine model based on $3 G$ cellular networks and agent-based technology. The block diagram of the respective frame 
work of the proposed model is depicted in Fig. 1. The proposed model is divided into the following units including patient unit, doctors unit, patient health record (PHR) database unit, technician unit and Social worker unit. Each of the modules is now detailed in the following section.

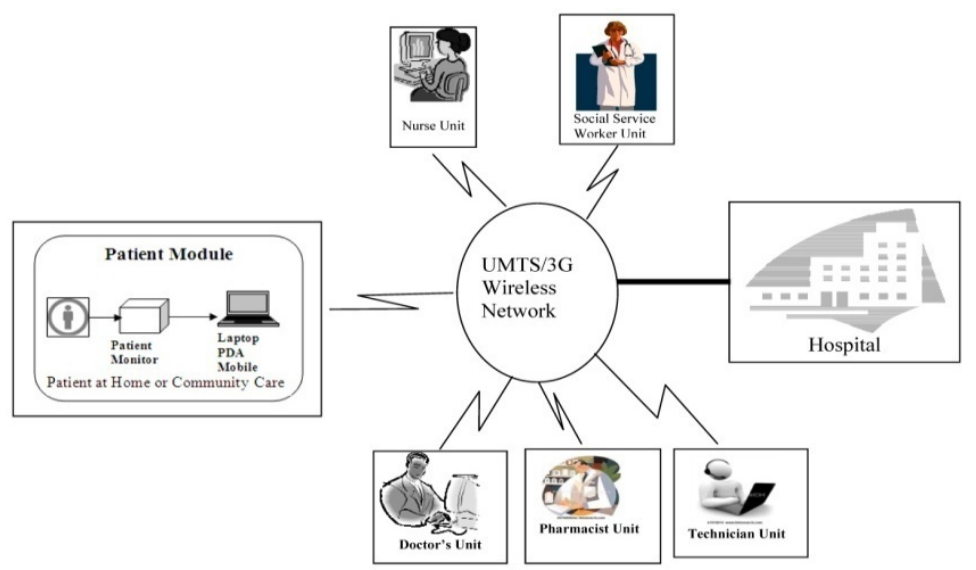

Fig. 1. Overview of the proposed System

\subsection{Patient Unit}

The patient unit which may be located in an ambulance, home or rural health care centres in remote areas or any other outdoor environment is implemented either on a laptop, desktop PC, mobile phone, or note book depending on the availability. Patient units which consist of vital sign monitors connected with computers/mobile phone collect and process vital medical data about patients, and transmit them through a $3 \mathrm{G}$ wireless link to the specialist unit which is connected to either a desktop computer within the existing hospital network or a mobile phone with $3 \mathrm{G}$ access capability at the physician's disposal. This enables the physicians to access the collected patient's data in a real-time mode or store-and-forward mode. After the measurement session, the acquired data can be transmitted to a database for later review and storage. For a vital signal acquisition, widely available off-the-shelf vital signal monitors will be used.

\subsection{Doctors Unit}

The doctors unit which may be located in a hospital, home or the outdoor environment, can be implemented like patients unit mentioned in Section 3.1 on a laptop, desk PC, mobile phone, or notebook. The specialist's or doctor's unit which is connected to either a fixed computer within an existing hospital network or a mobile phone with 3G access capability at the physician's hand receives the patient's medical data and analyses these data to provide the patients with meaningful information. As mentioned in Section 3.1, the doctors can access the collected patient's data in a RT 
mode or store-and-forward mode. If necessary the doctors can initiate real time video conferencing session with the patient unit or with other doctors.

\subsection{Patient Health Record Database Unit}

All the biological signals and patient's data are stored in the patient health record (PHR) database unit. These data are periodically sent from the patient unit to the PHR. This unit generates the alerts to the doctors for the emergency conditions either by an alert message from the patient unit or by analysing the patient data if it finds out some critical problem.

\subsection{Technician Unit}

The technician unit keeps track of the device error alert messages from the patient units.

\subsection{Social Worker Unit}

Integration with social services will often be necessary as a significant number of elderly people are also frail and require daily support, or suffer from dementia. Social Services can also be required to oversee the environmental issues and social alarms, and ideally these should be integrated with medical monitoring, as similar patient groups are involved.

\subsection{Pharmacist Unit}

The pharmacist unit will reside in a hospital or drug store or outdoor environment. This unit receives and monitors the medications for a patient and the outcome or results of applying the medicines. It operates through a web interface.

\section{Implementation}

The proposed model will provide the following RT/NRT services/operations between its various functional units as text file, image, audio, chat, whiteboard and web as shown in Fig. 2.

This system will follow a multi-collaborative design [8] and implement new RT multimedia features available for $3 \mathrm{G}$ wireless networks. Session Initiation Protocol (SIP) is chosen as the service control protocol along with messages defined specifically for the Internet Multimedia system (IMS) by the 3rd Generation Partnership Project (3GPP). Communication between the patient unit and one or more specialist units will take place in the form of multi-collaborative sessions through various network environments supporting various multimedia traffics. The conference model chosen is the one used in [8], [14] that consists of a central unit (CU) which is responsible for maintaining integration between different units. During the conference, each participant unit will have dialog established with the CU and the CU will ensure the media streams used in the conference are available to the appropriate users. The signals and the session management are detailed in the next section. 


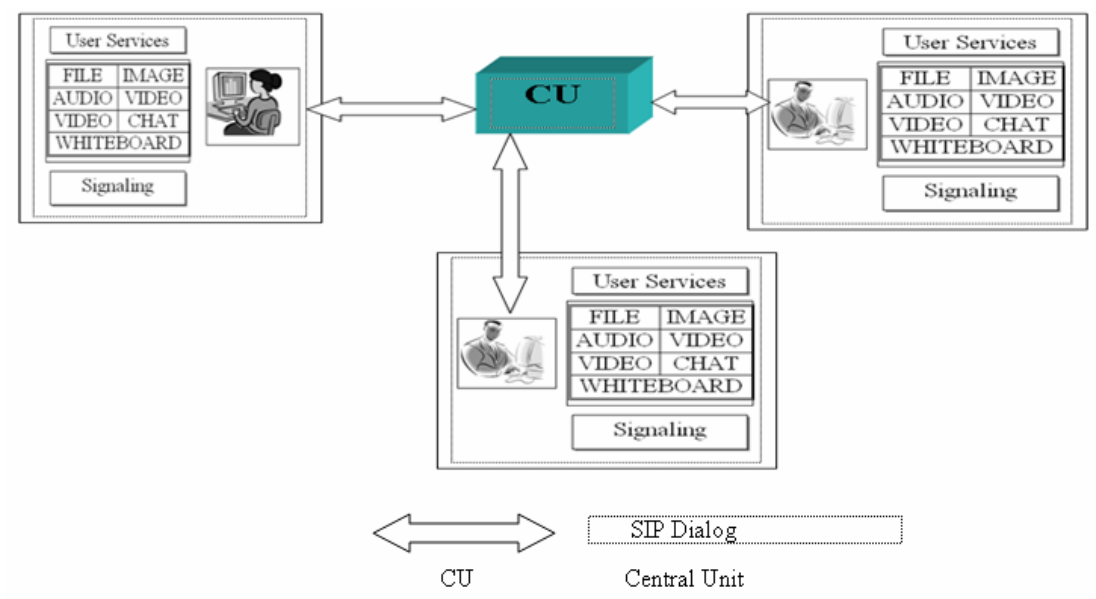

Fig. 2. Systems user services architecture

\subsection{Signaling and Session Management}

The SIP protocol performs signaling in the conference system by first initiating a SIP dialog with the CU. Different services are described by the use of Session Description Protocol (SDP) messages. To establish and maintain the SIP dialogs, the each unit involved in the conference is assigned a SIP user agent (UA) with a slightly modified version in CU to allow multiple dialogs concurrently.

Conference establishment, maintenance and termination are performed by the exchange of SIP messages between the conference participants. When a user enters the conference it first establishes a SIP dialog with the CU. Within the conference the different users exchange SIP messages between themselves and with the CU to vary conference characteristics and to allow for the management of the conference system. Similarly to conference joining, when a user wants to leave the conference it exchanges necessary messages with the $\mathrm{CU}$ for this purpose. SIP messages also carry the SDP messages which describe various medical user services. Since the CU may come to failure the users are also allowed to establish point to point sessions between them.

\subsection{User Services Management}

The user services in this proposed system is associated with one or more information which are going to be shared in the system. The system has services to share audio, video, still images, medical data (e.g., ECG, SPO2, blood sugar etc.), chat, electronic whiteboard and web service to access information retained in the database. There are also services for exchanging control information. Each of the medical information is associated with a user service. Each user service uses a transport according to their needs. The real-time services such as audio, video, medical data use the real-time protocol (RTP) whereas NRT services use the transmission control protocol (TCP). 


\subsection{Application Management}

The information generated by each service is first received by the CU which then forwards this information to the destination unit and in the way defined by the application management service by means of control messages. The CU forwards the information it receives but first mixes all the audio signals and sends a unique signal to each user. In case of video signals only one signal is sent to all the entities at given moment.

The user interface will be developed using J2SE \& J2ME development tools [4]. For implementation purposes, Oracle or SQL server will be used because they support data mining techniques such as clustering, decision tree [4].

\section{Multi-agent Based Software Model}

Providing effective and intelligent monitoring and medical care to the patients in a timely manner, and at the same time providing the doctors with decision support system which will enable them to more efficient with their tasks in the telemedicine system is of paramount importance. This will also ensure frequent patient-doctor interaction to maximize the system's utility. Towards this goal, it is necessary to develop intelligent management software for the system. A very good solution to the management software issue is the intelligent software agent [9-10], [15] which is capable of acting exactly in order to accomplish tasks with some reasoning or planning. To support telemedicine system efficiently it is necessary that the following requirements are met by the software system: (a) anticipating the information need for the users and delivering it to the user in a periodic and/or timely manner, (b) providing support for communication, coordination and cooperation among the various entities in the system such as doctors, nurses, patients and relatives, pharmacists, technicians, social service workers who are involved in the process of maintaining and delivering the health care services, (c) providing support for collaborative decision making among various entities involved in this health care system distributed information and knowledge sources, (d) relieving the people involved in the system from management of routine tasks, and (e) contributing in the automation process of the overall system [16].

The model designed especially for the $3 \mathrm{G}$ based telemedicine framework which depicted in Fig. 3 is based on the models in [9], [16]. This proposal aims at designing software agents (An intelligent agent is defined as one that is capable of flexible autonomous action to meet its design objectives [17]) which will work on behalf of human agents with similar characteristics. In other words, our solution delegates daily routine tasks performed by human agents to software agents. In this new approach, each actor is assigned a personalized software agent who acts as his personal human agent [9].

The software model includes an agent for each of these entities: patients, nurses, doctors (general or specialist), pharmacist and technician. These entities communicate with each other through the $3 \mathrm{G}$ network. Therefore, there are 7 different types of software agents in our model. They are Patient Agent (PA), Doctor Agent (DA), 


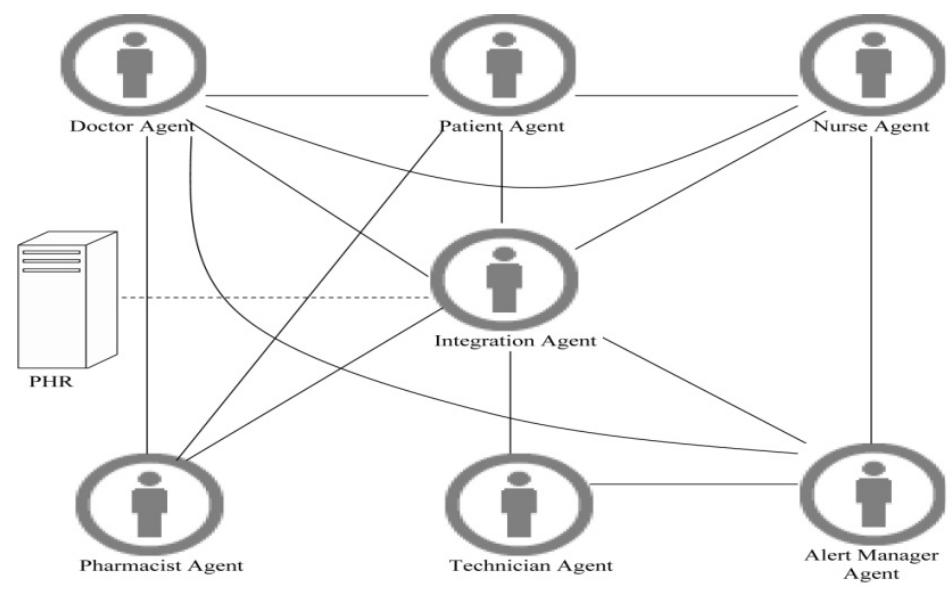

Fig. 3. Software model for advanced telemedicine framework

Nurse Agent (NA), Pharmacist Agent (PHA), Technician Agent (TA), Alert Manager Agent (AMA), and Integration Agent (IA). The PA is capable of carrying patient's data and to move the data to Patient Health Record (PHR) server where it interacts with the IA and requests IA to submit the data for the appropriate patient record. PA can also retrieve the patient's medical history records from the PHR database through the IA. PA also monitors vital signals received from the various patient side equipments and is capable of determining whether an alarm is false or real one and subsequently can forward the alert to appropriate agent (NA, DA, AMA). The IA has the ability to detect if the patient's situation is critical based on the medical data and predefined criteria, and it immediately sends an alarm to the NA which in turns notifies the nurse when the patient's medical data is abnormal. Simultaneously IA also sends the alarm notification to the AMA which keeps track of the alerts in the system. If the patient's situation becomes critical below some threshold criteria and the nurse is unable to make a decision, NA automatically forwards the alarm to the DA with a notification to the AMA. NA also provides the nurse with the summary of information of the patients associated with the nurse. The DA interacts with PA to get the required data and make decisions accordingly. DA keeps track of the patients currently under the doctor's supervision, and also provides the doctor with summary of his patients' conditions. It also keeps track of the alert messages from various PA sorts and presents them to the doctor in the order of urgency. Similarly, when the PA or DA receives device failure alert it will immediately forward the alert to the AMA and subsequently to TA where TA provides a notification to the technician. TA also keeps track of the device alerts and summarizes and presents them to the technician in some order. AMA monitors and keeps track of the alerts between different agents. The IA plays the central role and manages the total agent based architecture. It also keeps control of the PHR database. The PHA keeps tracks of the medication from the DA to PA. Whenever PA sets an alarm of medicine need to the PHA and PHA responses and then discards the alarm from its repository. Furthermore, NA, PA, DA can interact with IA and PHR at anytime with real time responses. With the agent based model presented, medical services can be provided instantly, remotely with convenience. 


\section{Conclusions}

This paper has presented an advanced $3 \mathrm{G}$ network and agent-oriented approach to developing automatic architecture for a generic telemedicine system to address the ever increasing complexity in next-generation telehealth systems. The proposed system would provide flexible way of delivering care to patients than is currently available in a number of healthcare scenarios or service platforms in rural, remote and emergency settings as well as in ambulance and home care. Further works are in progress to develop a prototype and to test the system under realistic scenarios.

\section{References}

1. Kyriacou, E., Pavlopoulos, S., Berler, A., Neophytou, M., Bourka, A., Georgoulas, A., Anagnostaki, A., Karayiannis, D., Schizas, C., Pattichis, C., Andreou, A., Koutsouris, D.: Multi-purpose HealthCare Telemedicine Systems with mobile communication link support. Biomedical Engineering Online 2(7). BioMed Central Ltd. (2003)

2. Kyriacou, E., Pattichis, M.S., Pattchis, C.S., Panayides, A., Pitsillides, A.: m-Health eEmergency Systems: Current Status and Future Directions. IEEE Antennas and Propagation Magazine 49(1), 216-231 (2007)

3. Leijdekkers, P., Gay, V.: Personal Heart Monitoring System Using Smart Phones To Detect Life Threatening Arrhythmias. In: 19th IEEE International Symposium on Computer-Based Medical Systems, Salt Lake City, Utah, pp. 157-164 (2006)

4. Sufi, F., Fang, V., Mahmoud, S.S., Cosic, I.: A Mobile Phone Based Intelligent Telemonitoring Platform. In: 3rd IEEE-EMBS International Summer School and Symposium on Medical Devices and Biosensors, Cambridge, MA, pp. 101-104 (2006)

5. Zhang, P., Kogure, Y., Matsuoka, H., Akutagawa, M., Kinouchi, Y., Zhang, Q.: A Remote Patient Monitoring System Using a Java-enabled 3G Mobile Phone. In: 29th Annual Conference of the IEEE EMBS, Lyon, pp. 3713-3716 (2007)

6. Chu, Y., Ganz, A.: A Mobile Teletrauma System Using 3G Networks. IEEE Trans. on Information Technology in Biomedicine 8(4), 456-462 (2004)

7. Kogure, Y., Matsuoka, H., Kinouchi, Y., Akutagawa, M.: The Development of a Remote Patient Monitoring System using Java-enabled Mobile Phones. In: 27th Annual Conference of IEEE Engineering in Medicine and Biology, pp. 2157-2160 (2005)

8. Navarro, E.A.V., Mas, J.R., Navajas, J.F.: Enhanced 3G-Based m-Health System. In: IEEE Eurocon 2005, pp. 1332-1335. BioMed. Central Ltd. (2005)

9. Nguyen, M.T., Fuhrer, P., Rocha, J.P.: Enhancing E-Health Information Systems with Agent Technology. Int. J. of Telemedicine and Application (2009)

10. Chan, V., Ray, P., Parameswaran, N.: Mobile e-Health monitoring: an agent-based approach. Comm. IET 2(2), 223-230 (2008)

11. Figueredo, M.V.M., Dias, J.S.: Mobile Telemedicine System for Home Care and Patient Monitoring. In: 26th Annual Conference of the IEEE EMBS, San Francisco, CA, pp. 3387-3390 (2004)

12. Istepanian, R.H., Laxminarayan, S., Pattichis, C.S.: M-Health: Emerging Mobile Health Systems. Springer, New York (2006)

13. Sandler, D.: A Call to needle times after acute myocardial infraction. BMJ (1999) 
14. Rosenberg, J.: A Framework for Conferencing with the Session Initiation Protocol. Internet draft (2004)

15. Nwana, H.: Software Agents: An Overview. The Knowledge Engineering Review 11(3), 205-244 (1996)

16. Liu, P.R., Meng, M.Q.H., Tong, F.F.L., Chen, X.J., Liu, P.X.: A $3 G$ based Network Solution to the Telehealthcare Robotic System. In: 6th World Congress on Intelligent Control and Automation, pp. 381-385. IEEE Press, Dalian (2006)

17. Chan, V.: 'Mobile health monitoring'. Thesis Presented in the University of New South Wales (2006) 